\title{
Fixation d'azote par le soja vietnamien (Glycine max) cv DH4 inoculé par Bradyrhizobium japonicum USDA 138 (G3)
}

\author{
C Thi Manh 1, JJ Drevon 2, ML Champigny 1* \\ ${ }^{1}$ Laboratoire de Photosynthèse et Métabolisme (URA D1128), bât 430, université Paris-Sud, F91405 Orsay cedex; \\ 2 INRAM, laboratoire Symbiotes des racines, 1, place Pierre-Viala, F34060 Montpellier cedex, France
}

(Reçu le 20 janvier 1993; accepté le 31 mars 1993)

\begin{abstract}
Résumé - Les quatre associations symbiotiques entre, d'une part, les sojas (Glycine max L) cv DH4 (soja vietnamien) et 171-16 et, d'autre part, les rhizobiums SMX11 (originaire de la vallée du Mékong) à croissance rapide et G3 (Bradyrhizobium japonicum USDA 138) à croissance lente sont cultivées sur milieu liquide à pH 6,3 dépourvu d'azote minéral. Les plantes dont les nodosités sont déjà en place et actives sont transférées à $30 \mathrm{j}$ sur des milieux à $\mathrm{pH} 5,3$ et 4,3. La réponse des 4 symbioses au pH du milieu est étudiée par comparaison, après 2 sem de la biomasse (quantité de matière sèche des plantes) et de la fixation de $\mathrm{N}_{2}$ ( $\mathrm{N}$ organique total des plantes) des plantes transférées à celles des plantes maintenues à $\mathrm{pH}$ 6,3. Trois types de réponses à l'acidification du milieu s'observent sans relation apparente avec les réponses individuelles des plantes et des bactéries au pH du milieu lors des cultures en conditions asymbiotiques: pour les symbioses de SMX11, le transfert en milieu acide n'a pas d'effet sur la fixation de $\mathrm{N}_{2}$ quel que soit l'hôte, tandis que pour les symbioses de G3, le transfert à pH 4,3 est inhibiteur avec 171-16, et stimulateur avec $\mathrm{DH}$ 4. Les sojas 171-16 et $\mathrm{DH} 4$, non inoculés, dépendants de $\mathrm{NO}_{3-}$, ont une croissance respectivement plus rapide et plus lente à $\mathrm{pH} 4,3$ qu'à $\mathrm{pH}$ 6,3. Enfin la croissance des rhizobiums est maximale respectivement à $\mathrm{pH} 5,3$ et 6,3 pour les souches G3 et SMXII. Les effets du pH du milieu ne sont donc en relation ni avec le génotype bactérien, ni avec le génotype de la plante pris isolément, mais dépendent vraisemblablement du contrôle génétique créé par les conditions de symbiose. G3 trouve ainsi, chez le soja DH4 et dans des conditions de milieu acide, les conditions d'environnement cellulaire les plus favorables à l'expression de sa capacité à fixer $\mathrm{N}_{2}$. II est conclu que la symbiose G3/DH4 non seulement tolère mais préfère le milieu acide en aquiculture. En conséquence, il apparaît souhaitable de tester au champ l'effet de l'inoculation du soja vietnamien DH4 avec la souche G3, en particulier dans le delta du Mékong où les sols sont très acides.
\end{abstract}

acidité / résistance / rhizobium / soja / symbiose

Summary - Dinitrogen fixation by the vietnamese soybean (Glycine max) cv DH4 inoculated by Bradyrhizobium japonicum USDA 138 (G3) is enhanced upon transfer to acid medium. Four symbiotic associations obtained by inoculation of soybean (Glycine $\max \mathrm{L}$ ) cV DH4 (from Vietnam) and 171-16, with Rhizobium SMX11, a fast-growing strain from the Mekong valley and G3 (Bradyrhizobium japonicum USDA 138), a slow-growing strain, were grown on $\mathrm{N}$-free solution at $\mathrm{pH}$ 6.3. Acid treatments were applied by transfer of 30-d-old plants with well-developed nodules onto solutions at $\mathrm{pH} 5.3$ and 4.3. The effect of $\mathrm{pH}$ was studied after 2 wk by measuring biomass (plant dry weight) and $\mathrm{N}_{2}$ fixation (organic $\mathrm{N}$ per plant). Three different responses to acid $\mathrm{pH}$ were observed with no apparent connection with individual responses to acid $\mathrm{pH}$ of plants and rhizobia when grown asymbiotically: SMX11 symbiosis was resistant to acid $\mathrm{pH}$ whatever the host soybean, while G3/171-16 symbiosis was sensitive to acid $\mathrm{pH}$, and G3/DH4 preferred $\mathrm{pH}$ 4.3. The non-inoculated soybeans 171-16 and $\mathrm{DH} 4$ cultured on $\mathrm{NO}_{3}$ grew respectively faster and more slowly at $\mathrm{pH} 4.3$ than at $\mathrm{pH}$ 6.3. Maximum growth rate of the free rhizobia was at $\mathrm{pH} 5.3$ and 6.3 for G3 and SMX11 respectively. Thus, the effects of growth $\mathrm{pH}$ have no relationship with the rhizobia or the plant genotype considered alone. They likely depend on the genetic control developed by the symbiotic conditions. When inoculated in soybean $\mathrm{DH} 4$ and grown on acid medium, G3 finds the most favorable cellular conditions for the expression of its $\mathrm{N}_{2}$ fixation capacity. The G3/DH4 symbiotic association not only tolerates but prefers growing on acid medium. In conclusion it appears advisable to test the effect of inoculation of the vietnamese soybean DH4 with the strain G3 in the field, especially in the Mekong delta where soils are known to be very acid.

* Correspondance et tirés à part 


\section{INTRODUCTION}

En plus de l'intérêt économique qu'elle représente par rapport à l'emploi d'engrais azotés, la symbiose soja-rhizobium a l'avantage de permettre dans le delta supérieur du Mékong l'obtention de rendements élevés de grains riches en protéines (Duong et al, 1984a). L'implantation de la culture du soja en sol tropical se heurte aux difficultés liées à l'acidité du sol, avec les caractéristiques chimiques (excès de $\mathrm{Mn}^{2+}$ et $\mathrm{Al}^{3+}$, manque de $\mathrm{K}^{+}, \mathrm{Ca}^{2+}, \mathrm{Mg}^{2+}$ ) et physiques (organisation structurale, hydromorphie) qui lui sont associées (Latham et al, 1984). Après plusieurs années de culture de riz, le sol ne contient plus de rhizobium nodulant le soja à cause de l'abaissement de son $\mathrm{pH}$ à 4,5-5,1 et le nombre de nodosités est très réduit (Duong et al, 1984a). Ce sont des facteurs limitants de la symbiose du soja avec Bradyrhizobium japonicum (Mengel et Kamprath, 1978) qui diminuent la production de protéines.

Plusieurs procédés ont été élaborés pour lutter contre cette difficulté : un remède au manque de bactéries peut être l'introduction dans les sols d'un engrais biologique (Nitragin Co, Wisconsin, USA), mélange complexe de plusieurs souches de Rhizobium japonicum, qui augmente la production des grains à l'hectare, leur teneur en protéines, et raccourcit le cycle de vie des plantes (Duong et al, 1984a; 1984b) ; l'addition, dans le sol, de cendres de tissus végétaux, s'est avérée un moyen efficace d'amélioration de la germination et de la croissance des sojas (Duong and Diep, 1986); un autre procédé d'amélioration, dont l'approche est décrite cidessous, repose, comme pour la culture de Trifolium subterraneum, en symbiose avec Rhizobium trifolii (Thornton et Davey, 1983), sur la sélection de variétés de sojas, et de souches de rhizobium capables de former des associations symbiotiques résistantes au milieu acide et de réaliser une fixation active de $\mathrm{N}_{2}$ permettant de bons rendements en protéines.

L'acidité des sols agit à différentes étapes du développement des Légumineuses en symbiose: multiplication et survie des bactéries dans la rhizosphère ; infection des racines avec formation des nodosités ; fixation de l'azote atmosphérique et développement des plantes (Lie, 1969). L'effet du $\mathrm{pH}$ sur la nodulation des Légumineuses a été très étudié (Munns et al, 1981). La nodulation est l'étape la plus sensible au pH acide (Evans et al, 1980). Puisqu'il est possible de protéger la germination, la nodulation et les pemiers temps de la croissance des plantes par l'addition au sol de cendres qui en relèvent le $\mathrm{pH}$, les recherches décrites ci-dessous ont considéré la sensibilité au $\mathrm{pH}$ acide, uniquement lors du transfert sur milieu de $\mathrm{pH} 5,3$ ou 4,3, d'associations symbiotiques préalablement établies à $\mathrm{pH} 6,3$, bien nodulées, et capables de fixer activement l'azote atmosphérique. Les partenaires de la symbiose, variétés de soja et souches de rhizobium, furent sélectionnés auparavant par comparaison de leur croissance en milieu de $\mathrm{pH} 4,3$ à 6,3 à l'état libre pour les bactéries et en présence de nitrates pour les sojas non nodulés. Les traitements des plantes inoculées sur milieux de différents $\mathrm{pH}$ sont faits en aquaculture. La réponse des associations symbiotiques considérées est faite par mesure de la biomasse (quantité de matière sèche des plantes) et de la fixation de $\mathrm{N}_{2}$ (quantité de $\mathrm{N}$ organique des plantes).

\section{MATÉRIEL ET MÉTHODES}

\section{Matériel végétal}

Les sojas (Glycine max L) testés quant à leur sensibilité au $\mathrm{pH}$ du milieu appartiennent aux variétés Kingsoy, fournies par Northrup King Semences (31790 Saint-Sauveur, France), Alaric, Swift, Weber, 171-16 originaires du domaine de I'INRA à Melgueil (Montpellier), et $\mathrm{DH} 4$, en provenance de l'université de Can Tho (Viêt-Nam).

Les souches de rhizobium ont des origines diverses, précisées dans le tableau I. La classification de SMX11 originaire du Sud Viêt-Nam est délicate (Chen et al, 1988). De croissance rapide comme les Sinorhizobium fredii isolés des sols tropicaux et subtropicaux (Scholla et Elkan, 1984), elle ne provoque pas l'acidification du milieu caractéristique de ces souches (Young et al, 1988), mais son alcalinisation (Cleyet-Marel, 1987), ce qui est typique des Bradyrhizobium à croissance lente (Keyser et al, 1982).

\section{Cultures}

Les cultures de bactéries sont faites sur milieu YEM

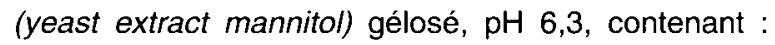
$\mathrm{KH}_{2} \mathrm{PO}_{4} 0,5 \mathrm{~g}, \mathrm{MgSO}_{4} 7 \mathrm{H}_{2} \mathrm{O} 0,2 \mathrm{~g}, \mathrm{NaCl} 0,2 \mathrm{~g}$, $\mathrm{CaCO}_{3} 0,1 \mathrm{~g}, \mathrm{CaSO}_{4} 2 \mathrm{H}_{2} \mathrm{O} 0,1 \mathrm{~g}$, et $10 \mathrm{~g}$ de mannitol, $0,5 \mathrm{~g}$ de glutamate de $\mathrm{Na}, 0,5 \mathrm{~g}$ d'extrait de levure, $15 \mathrm{~g}$ d'agar/l (Robertson et Taylor, 1973). Les souches sont conservées sur milieu gélosé à $+4^{\circ} \mathrm{C}$. Elles sont entretenues par repiquage tous les 2 mois. Avant inoculation des sojas, les bactéries sont repiquées stérile- 
Tableau I. Origine et provenance des souches de rhizobium.

\begin{tabular}{lll} 
Souche & Origine & Provenance \\
\hline G3 (USDA 138) & & \\
G49 (IARI SB16) & USA, Beltsville & INRA, Dijon \\
USDA 110 & Inde & INRA, Montpellier \\
G9 & USA & INRA, Montpellier \\
SMX11 & Sud Vietnam & INRA, Montpellier \\
SMGS1 (SA1 CIRAD) & Sud Vietnam & INRA, Montpellier \\
SMA, Montpellier
\end{tabular}

ment sur milieu YEM liquide identique à celui qui est décrit plus haut, excepté que la gélose en est absente. La culture des bactéries en suspension dans le milieu liquide dure $4 \mathrm{j}$ à $28-30^{\circ} \mathrm{C}$ sur un agitateur (120 oscillations`min ${ }^{-1}$ ). Les bactéries sont alors sédimentées par centrifugation à $4000 \mathrm{~g}$ pendant $10 \mathrm{~min}$, lavées à l'eau distillée stérile et remises en suspension dans l'eau stérile à la densité de $10^{9}$ cellules $/ \mathrm{ml}$, correspondant à une densité optique de $0,8-1,0$ à $620 \mathrm{~nm}$ sous une épaisseur de $1 \mathrm{~cm}$.

Les graines de soja sont stérilisées en surface par trempage pendant $15 \mathrm{~min}$ dans l'hypochlorite de calcium à $0,35 \%$, puis abondamment lavées à l'eau distillée. La germination a lieu à $28^{\circ} \mathrm{C}$, sur papier filtre imprégné de $\mathrm{CaSO}_{4} 0,1 \mathrm{mM}$. Après 4 à $5 \mathrm{j}$, les graines germées dont les radicules mesurent 8 à $10 \mathrm{~cm}$ sont sélectionnées pour le repiquage, précédé ou non de l'inoculation.

L'inoculation se fait par immersion des radicules pendant 30 min dans la suspension de rhizobium à $10^{9}$ cellules par $\mathrm{ml}$, préparée comme indiqué cidessus. Immédiatement après l'inoculation, les sojas sont plantés dans la vermiculite préalablement lavée à l'eau déminéralisée par électrodialyse, à raison de 3 plantes par pot de $3 \mathrm{I}$. Aucun arrosage n'est fait pendant les 4 premiers jours, afin de ne pas entraîner les bactéries par lessivage. Puis les plantes reçoivent tous les $2 \mathrm{j}$ jusqu'à l'âge de $30 \mathrm{j}$, une solution nutritive $(\mathrm{pH}$ 6,3) contenant $\mathrm{KH}_{2} \mathrm{PO}_{4} 0,75 \mathrm{mM}, \mathrm{K}_{2} \mathrm{HPO}_{4} 0,25 \mathrm{mM}$, $\mathrm{MgSO}_{4} 7 \mathrm{H}_{2} \mathrm{O} 2,5 \mathrm{mM}, \mathrm{NaCl} 0,2 \mathrm{mM}, \mathrm{K}_{2} \mathrm{SO}_{4} 5 \mathrm{mM}$, $\mathrm{CaCl}_{2} 2,5 \mathrm{mM}, 10 \mathrm{mg} . \mathrm{I}^{-1} \mathrm{du}$ complexe Fe-EDTA (Alcantar-Gonzales et al, 1990) et les oligo-éléments selon Arnon (1938).

Les plantes non inoculées sont également repiquées sur vermiculite après $4-5 \mathrm{j}$ de germination. Le milieu nutritif de $\mathrm{pH} 6,3$ a la même composition que le précédent, mais $\mathrm{CaCl}_{2} 2,5 \mathrm{mM}$ est remplacé par $\mathrm{Ca}$ $\left(\mathrm{NO}_{3}\right)_{2} 2,5 \mathrm{mM}$.

\section{Traitements par les milieux à $p H$ acide}

La croissance des bactéries dans le milieu YEM à pH 6,3 ou ajusté à $\mathrm{pH} 4,3$ et 5,3 par addition de $\mathrm{HCl} \mathrm{N}$ stérile est suivie par mesure de la densité optique à 620 $\mathrm{nm}$ pendant les 6 à 20 j qui suivent la mise en suspension des bactéries prélevées sur le milieu gélosé. Le
$\mathrm{pH}$ du milieu est ajusté à sa valeur d'origine toutes les 6 ou $12 \mathrm{~h}$. Les inoculums de départ sont identiques (contrôle par mesure de l'absorbance à $620 \mathrm{~nm}$ ). Le temps de génération est calculé pendant la phase exponentielle de croissance. Les résultats sont la moyenne de 4 cultures (tableau II).

Inoculés ou non, les sojas sont transplantés à l'âge de $30 \mathrm{j}$ de la vermiculite sur le milieu nutritif liquide de pH 6,3. Seule la moitié inférieure du chevelu racinaire est immergée dans la solution nutritive. Les nodosités qui sont situées sur la partie supérieure des racines restent à l'air. Après $48 \mathrm{~h}$ à $\mathrm{pH} 6,3$, le $\mathrm{pH}$ du milieu est abaissé à 5,3 et 4,3 , par addition de $\mathrm{HCl} \mathrm{N}$, ou conservé à $\mathrm{pH} 6,3$. Afin d'assurer aux cultures un $\mathrm{pH}$ constant, le milieu est renouvelé tous les jours. Les plantes sont prélevées pour analyse après $14 \mathrm{j}$ de traitement.

\section{Prélèvement des plantes, détermination de la biomasse et stabilisation du matériel végétal en fin d'expérience}

Les différents organes, nodosités, racines, tiges et feuilles de chaque plante sont prélevés et analysés

Tableau II. Temps de génération des souches de rhizobium G3, G49, SMGSI, G9, USDA 110, SMX11.

\begin{tabular}{lccc}
\hline \multirow{2}{*}{ Souches } & \multicolumn{3}{c}{$p H$ du milieu } \\
\cline { 2 - 4 } & 4,3 & 5,3 & 6,3 \\
\hline & & & \\
G3 & 600 & 30 & 42 \\
G49 & 686 & 31 & 44 \\
SMGSI & 600 & 32 & 42 \\
G9 & 437 & 44 & 31 \\
USDA 110 & 534 & 44 & 28 \\
SMX11 & 240 & 42 & 24 \\
\end{tabular}

La croissance est suivie par mesure de l'absorbance à 620 $\mathrm{nm}$ pendant $2 \mathrm{j}$ de culture sur milieux de $\mathrm{pH} 4,3 ; 5,3 ; 6,3$. Les résultats sont exprimés en $h$. Ils sont la moyenne des temps de génération de 4 cultures. 
Tableau III. Croissance des sojas, cv Kingsoy, 17116, Weber, Alaric, Swift, DH4, non symbiotiques, mesurée par la quantité de matière sèche totale des plantes après $30 \mathrm{j}$ de culture sur vermiculite $(\mathrm{pH} 6,3)$ suivis de $14 \mathrm{j}$ en aquiculture sur milieu de $\mathrm{pH} 4,3 ; 5,3$; 6,3 .

\begin{tabular}{|c|c|c|c|}
\hline \multirow[t]{3}{*}{ Sojas } & \multicolumn{3}{|c|}{$p H$ du milieu } \\
\hline & 4,3 & 5,3 & 6,3 \\
\hline & \multicolumn{3}{|c|}{ Matière sèche (mg/plante) } \\
\hline Kingsoy & Mort rapide & 1209 * & 923 \\
\hline $171-16$ & $1610^{*}$ & 1620 * & 1394 \\
\hline Weber & $1078^{*}$ & 1125 * & 1420 \\
\hline Alaric & 4006 & 4166 & 4394 \\
\hline Swift & 2781 & 2993 & 2986 \\
\hline $\mathrm{DH} 4$ & $1382^{*}$ & 1501 & 1631 \\
\hline
\end{tabular}

* Différences significatives au risque $5 \%$ par rapport à la culture de la même association sur milieu de $\mathrm{pH} 6,3 . n=4$.

séparément. Les résultats présentés sont la moyenne de 4 plantes.

Les tiges, feuilles et racines sont desséchées à l'étuve à $80^{\circ} \mathrm{C}$ sous courant d'air pendant $72 \mathrm{~h}$. La matière sèche est pesée dans des flacons tarés, broyée en poudre et conservée à $+4^{\circ} \mathrm{C}$ dans un dessicateur sous vide en présence de $\mathrm{CaCl}_{2}$ jusqu'à analyse. Immédiatement après leur excision des racines, les nodosités sont rapidement lavées à l'eau distillée à $+4^{\circ} \mathrm{C}$, puis séchées sur papier filtre et leur masse de matière fraîche est déterminée par pesée. Elles sont conservées dans l'azote liquide à $-80^{\circ} \mathrm{C}$ jusqu'à analyse.

\section{Dosage de l'azote organique total}

Des fractions aliquotes de la poudre sèche du matériel végétal, ou la totalité des nodosités de chaque plante, sont prélevées. La minéralisation est faite en présence d'acide sulfurique $36 \mathrm{~N}$ en présence du catalyseur à sélénium Dumazert, à $280^{\circ} \mathrm{C}$ sur un bain de sable. L'azote organique transformé en $\mathrm{NH}_{4}^{+}$, est coloré par le réactif de Nessler et dosé par colorimétrie avec lecture de la densité optique à $490 \mathrm{~nm}$ (Umbreit et al, 1964). La courbe standard est obtenue avec une solution d'oxalate d'ammonium de 4 à $40 \mu \mathrm{g}$ d'azote.

\section{Analyse statistique}

L'analyse de variance a été faite à l'intérieur de chaque échantillon. La comparaison des moyennes a été faite entre chaque plante prise isolément (tableau III) ou en association symbiotique (tableaux IV, V, VI) sur milieu à $\mathrm{pH}$ acide et la même plante ou association symbiotique sur milieu de $\mathrm{pH}$ 6,3, prise comme témoin. L'utilisation de la table de Fisher et Yates a permis de déterminer les différences significatives (Schwartz, 1963).

\section{RÉSULTATS}

\section{Effet du pH sur la croissance des rhizobiums et des sojas non symbiotiques}

$\grave{A} \mathrm{pH} 4,3$ la croissance de toutes les souches de rhizobium étudiées est inhibée (tableau II). Les souches G3, G49 et SMGS1 ont des temps de génération plus courts à $\mathrm{pH} 5,3$ qu'à $\mathrm{pH} 6,3$ alors

Tableau IV. Croissance des sojas DH4 et 171-16, inoculés par les rhizobium G3 ou SMX11, mesurée par la quantité de matière sèche totale des plantes après 30 jours de culture sur vermiculite $(\mathrm{pH} 6,3)$ suivis de $14 \mathrm{j}$ en aquiculture sur milieu de $\mathrm{pH} 4,3 ; 5,3 ; 6,3$.

$\begin{array}{cc}\text { Rhizobium/soja } & \text { Vermiculite } \\ 30 j & p H 6,3\end{array}$

Aquiculture, les $14 j$ suivants pH du milieu

5,3
MS acquise (mg/plante)

$\begin{array}{lrccc} & & & \\ \text { SMX11/DH4 } & 1208 & 640 & 679 & 664 \\ \text { G3/DH4 } & 976 & 916^{*} & 339 & 487 \\ \text { SMX11/171.16 } & 788 & 583 & 450 & 395 \\ \text { G3/171.16 } & 1242 & 74^{*} & \text { nd } & 570\end{array}$

\footnotetext{
* Différences significatives au risque 5\% par rapport à la culture de la même association sur milieu de pH 6,3. $n=4$.
} 
Tableau V. N organique des sojas DH4 et 171-16, inoculés par les rhizobium G3 ou SMX11, après 30 j de culture sur vermiculite $(\mathrm{pH} 6,3)$ et quantité acquise pendant les 14 j suivants en aquiculture sur milieu de $\mathrm{pH} 4,3 ; 5,3 ; 6,3$.

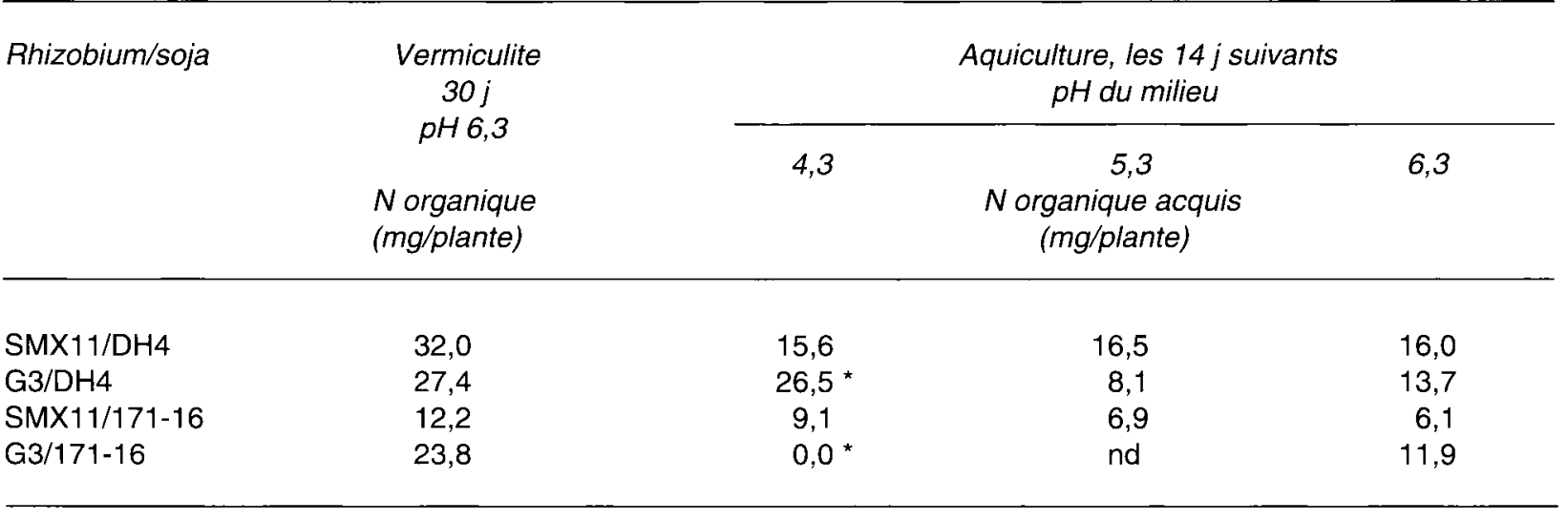

* Différences significatives au risque 5\% par rapport à la culture de la même association sur milieu de pH 6,3. $n=4$. nd : mesure non déterminée.

Tableau VI. Nombre, matière fraîche des nodosités de sojas DH4 et SMX11 inoculés par les rhizobium G3 ou SMX11, après $30 \mathrm{j}$ de culture sur vermiculite $(\mathrm{pH} 6,3)$ suivis de $15 \mathrm{j}$ en aquiculture sur milieu de $\mathrm{pH} 4,3 ; 5,3 ; 6,3$.

\begin{tabular}{|c|c|c|c|c|c|c|}
\hline \multirow{4}{*}{ Rhizobium/soja } & \multicolumn{6}{|c|}{ Nodosités } \\
\hline & \multicolumn{3}{|c|}{ Nb par plante } & \multicolumn{3}{|c|}{ Matière fraiche (mg/plante) } \\
\hline & \multicolumn{3}{|c|}{$p H d u$ milieu } & \multicolumn{3}{|c|}{$p H$ du milieu } \\
\hline & 4,3 & 5,3 & 6,3 & 4,3 & 5,3 & 6,3 \\
\hline $\mathrm{SMX11/DH4}$ & $50^{*}$ & $68^{*}$ & 83 & 688 & 817 & 799 \\
\hline G3/DH4 & $51^{*}$ & 44 & 30 & 768 * & 503 & 510 \\
\hline $\operatorname{SMX} 11 / 171.16$ & 60 & 59 & 60 & 537 & 476 & 472 \\
\hline G3/171.16 & $15^{*}$ & nd & 38 & $190^{\star}$ & nd & 517 \\
\hline
\end{tabular}

* Différences significatives au risque $5 \%$ par rapport à la culture de la même association sur milieu à pH $6,3 . n=4$. nd : mesure non déterminée.

que, pour SMX11, USDA 110 et G9, c'est à $\mathrm{pH}$ 6,3 que la croissance est la plus rapide. C'est alors SMX11, souche à croissance rapide d'origine vietnamienne, qui a le temps de génération le plus court. Cela est en accord avec les observations de Cleyet-Marel (1987) qui la caractérise comme une souche à croissance rapide.

La croissance sur $\mathrm{NO}_{3}-5 \mathrm{mM}$ des 6 sojas nodulés permet de distinguer 4 types de réponses au $\mathrm{pH}$ du milieu: 1) Kingsoy meurt à $\mathrm{pH} 4,3$; 2) Weber et $\mathrm{DH} 4$ sont sensibles à l'acidité du milieu; 3) 171-16 préfère les milieux acides; 4) Alaric et Swift sont indifférents au $\mathrm{pH}$ du milieu (tableau III).

\section{Effet du pH sur la croissance et la nutrition azotée de sojas symbiotiques}

Les associations symbiotiques choisies pour cette étude sont les combinaisons de 2 bactéries, $B$ japonicum G3, originaire des USA, se multipliant le plus activement à $\mathrm{pH} \mathrm{5,3} \mathrm{et,} \mathrm{pour}$ comparaison, la souche SMX11 d'origine vietnamienne, se multipliant le plus activement à $\mathrm{pH}$ 6,3 , avec 2 variétés de sojas, $\mathrm{DH} 4$ d'origine vietnamienne, sensible au $\mathrm{pH}$ acide et 171-16 préférant les milieux acides. L'une des 4 associations, SMX11/DH4, est donc uniquement constituée d'organismes originaires du Sud Viêt-Nam. 
La biomasse produite pendant les $30 \mathrm{j}$ à $\mathrm{pH}$ 6,3 , qui précèdent le transfert en $\mathrm{pH}$ acide, classe les associations symbiotiques dans l'ordre décroissant, G3/171-16, SMX11/DH4, G3/

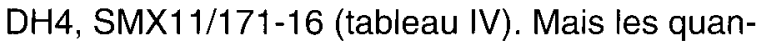
tités d'azote organique par plante (feuilles + tiges + racines), représentatives de la quantité d'azote atmosphérique assimilé, sont plus élevées chez SMX11/DH4 et G3/DH4 que chez G3/ 171-16 et $S M X 11 / 171-16$, ce qui suggère que la fixation de $\mathrm{N}_{2}$ est favorisée par la symbiose avec le soja DH4 (tableau V).

En $14 \mathrm{j}$ de traitement en aquiculture à $\mathrm{pH} 4,3$, 5,3 ou 6,3 , la production de biomasse du soja $\mathrm{DH} 4$ inoculé avec la souche SMX11 est indépendant $\mathrm{du} \mathrm{pH}$, alors qu'elle est stimulée par le transfert à $\mathrm{pH} 4,3$ pour les plantes inoculées avec la souche G3. Chez le soja 171-16, le transfert à $\mathrm{pH}$ acide n'a pas d'effet sur les plantes inoculées avec SMX11, tandis qu'il diminue la production de biomasse de celles qui sont inoculées avec G3 (tableau IV).

Les accumulations d'azote organique varient toujours dans le même sens que les productions de matière sèche, sous l'effet du pH du milieu, puisque les teneurs ne varient pas de façon significative (tableau $V$ ). Le transfert en milieu acide n'a pas d'effet avec SMX11, quelle que soit la plante-hôte, alors que ce traitement diminue significativement l'accumulation d'azote chez G3/171-16, et la stimule significativement chez G3/DH4.

\section{Effet du pH sur la nodulation et la fixation d'azote}

Chez le soja DH4 inoculé avec SMX11, le nombre de nodosités par plante est significativement plus faible à pH 5,3 et 4,3 qu'à pH 6,3 alors que la masse de matière fraîche totale des nodosités est la même (tableau VI). Ainsi, les nodosités sont-elles moins nombreuses mais plus grosses lorsque le système symbiotique SMX11/ $\mathrm{DH} 4$ est sur milieu acide. Chez les associations $\mathrm{G} 3 / \mathrm{DH} 4$, c'est à la fois le nombre et la masse de matière fraîche des nodosités qui s'accroissent significativement plus à pH 4,3 qu'à pH 5,3 et 6,3 pendant les $14 \mathrm{j}$ de culture.

Les nodosités développées sur le système racinaire du soja $171-16$ sont plus nombreuses lorsqu'il est inoculé par SMX11 que par G3, mais la masse de matière fraîche totale n'est pas significativement différente. Ce n'est que chez G3/
Tableau VII. Sojas DH4 et SMX11 inoculés par les rhizobium G3 ou SMX11, cultivés pendant 30 j sur vermiculite $(\mathrm{pH} 6,3)$ suivis de $14 \mathrm{j}$ en aquiculture sur milieu de $\mathrm{pH} 4,3 ; 5,3 ; 6,3$.

\begin{tabular}{lccc} 
Rhizobium/soja & \multicolumn{3}{c}{$\mathrm{N}_{2}$ fixé (mg/g MF de nodosités) } \\
\cline { 2 - 4 } & \multicolumn{3}{c}{$p H$ du milieu } \\
& 4,3 & 5,3 & 6,3 \\
& & & \\
& & & \\
SMX11/DH4 & 22,7 & 20,2 & 20,1 \\
G3/DH4 & $34,6^{*}$ & 16,1 & 26,8 \\
SMX11/171.16 & 17,0 & 14,5 & 12,9 \\
G3/171.16 & $0,0^{*}$ & nd & 23,1 \\
& \multicolumn{3}{c}{} \\
\hline
\end{tabular}

Quantité de $\mathrm{N}_{2}$ fixée par $\mathrm{g}$ de matière fraîche de nodosités, pendant les $14 \mathrm{j}$ en aquiculture. ${ }^{*}$ Différences significatives au risque $5 \%$ par rapport à la culture de la même association sur milieu de $\mathrm{pH} 6,3$. $n=4$. nd : mesure non déterminée.

171-16 que la culture pendant $14 \mathrm{j}$ à $\mathrm{pH} 4,3$ a significativement ralenti le développement en nombre et en masse des nodosités (tableau VI).

La capacité de fixation de $\mathrm{N}_{2}$ des nodosités, exprimée par la quantité d'N organique acquis par la plante entière en 14 jours de traitement rapportée à l'unité de matière fraîche des nodosités, est significativement stimulée par l'acidification du milieu chez G3/DH4 et inhibée chez G3/ 171-16 (tableau VII). Elle n'est pas affectée dans les deux cas de symbiose avec SMX11.

\section{DISCUSSION}

L'acidité du milieu $(\mathrm{pH} \mathrm{4,3)} \mathrm{inhibe} \mathrm{la} \mathrm{croissance}$ de toutes les souches de rhizobiums qui ont fait l'objet de cette étude (tableau I). Cet effet est conforme aux observations agronomiques qui relatent la disparition des rhizobiums inoculant le soja dans les sols à $\mathrm{pH} 4,5-5$ (Duong et al, 1984a ; 1984b). Nos observations permettent de distinguer 2 groupes de bactéries : celles qui préfèrent le milieu à $\mathrm{pH} 5,3$ et celles qui préfèrent le milieu à $\mathrm{pH} 6,3$, ce qui est le cas de la souche d'origine vietnamienne. Chez les sojas non inoculés et cultivés sur $\mathrm{NO}_{3}-$ tous les degrés sont observés, depuis le caractère léthal jusqu'au caractère favorable du milieu à $\mathrm{pH}$ acide.

Les 4 associations symbiotiques choisies pour la présente étude combinent un rhizobium à croissance lente et préférant le milieu de $\mathrm{pH} 5,3$ 
(G3), un rhizobium à croissance rapide, originaire du Sud Viêt-Nam et sensible à l'acidité (SMX11), à un soja préférant le milieu acide (171-16) et un

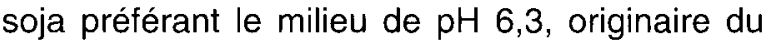
Sud Viêt-Nam (DH4). La biomasse exprimée par la quantité de matière sèche des plantes de $30 \mathrm{j}$, inoculées et toujours cultivées sur milieu de $\mathrm{pH}$ 6,3 , n'est que légèrement inférieure à celle des plantes de même âge, mais alimentées par $\mathrm{NO}_{3}-$ $5 \mathrm{mM}$ au même pH (tableaux III et IV). II est intéressant de comparer l'efficacité des bactéries fixatrices d'azote dans les plantes hôtes, exprimée par le rapport entre la quantité d'azote organique acquis par les plantes et la masse de matière fraîche des nodosités. Elle concerne l'interaction du rhizobium avec le génotype des plantes : à pH 6,3, avec le soja 171-16 (originaire des régions tempérées) comme avec $\mathrm{DH} 4$ (originaire des régions tropicales), c'est la souche G3 qui est la plus efficace (tableau VII).

Les effets du transfert des plantes en symbiose en conditions de milieu acide sur la production de biomasse ou la fixation d'azote n'ont aucune relation apparente avec les réponses individuelles des plantes et des bactéries au $\mathrm{pH}$ du milieu. La réponse au $\mathrm{pH}$ du milieu ne peut donc pas être déduite du comportement des partenaires isolés, mais strictement du comportement de l'association symbiotique. Les nodosités ne sont pas en contact direct avec le milieu et l'influence de celui-ci sur leurs activités ne peut se faire que par l'intermédiaire des échanges solplante, des translocations entre organes, des modifications du métabolisme cellulaire et des informations génétiques. Trois types de réponses à l'acidification du milieu sont observées : tolérance lorsque le symbiote est $S M \times 11$ et quel que soit I'hôte, sensibilité au milieu acide pour G3/ 171-16, préférence pour le milieu de $\mathrm{pH} \mathrm{4,3}$ pour G3/DH4. G3 trouve donc chez le soja $\mathrm{DH} 4$ et dans des conditions de milieu acide les conditions d'environnement cellulaire les plus favorables à l'expression de sa capacité à fixer $\mathrm{N}_{2}$. Lorsque les nodosités sont déjà mises en place et actives, les effets du $\mathrm{pH}$ du milieu sont en relation non pas avec le génotype bactérien ni avec le génotype de la plante, mais dépendent vraisemblablement du contrôle génétique créé par les conditions de symbiose.

\section{CONCLUSION}

Cette étude montre que la création d'une nouvelle association plante/rhizobium permet l'appa- rition de nouvelles propriétés qui lui sont propres et sans relation apparente avec les comportements des partenaires isolés. II serait intéressant d'approfondir les caractères agronomiques de la symbiose entre le soja vietnamien $\mathrm{DH} 4$ et B japonicum G3 (USDA 311 B 138) en plus de la préférence pour le milieu acide. Au cours des essais en aquiculture, les effets toxiques secondaires de l'acidité sont éliminés (solubilisation des ions toxiques $\mathrm{Al}^{3+}$ et $\mathrm{Mn}^{2+}$, pertes en cations $\mathrm{K}^{+}, \mathrm{Ca}^{2+}, \mathrm{Mg}^{2+}$, fixation d'anions $\mathrm{SO}_{4}{ }^{2-}$, $\mathrm{SO}_{4}{ }^{2-}$, etc; Sanchez et al, 1983). Il est par conséquent nécessaire d'élargir la connaissance du comportement de l'association symbiotique aux conditions de culture au champ avant de décider si l'inoculation du soja vietnamien $\mathrm{DH} 4$ par $B$ japonicum G3 (USDA 311 B 138) peut être préconisée.

\section{RÉFÉRENCES}

Alcantar-Gonzales G, Moyse A, Bismuth E, Champigny ML (1990) Effect of nitrate on acetylene reduction and the activities of some enzymes of nitrogen and carbon metabolism, involved in nitrogen fixation in the root nodules of soybean. agronomie 10, 847-855

Arnon DI (1938) Microelements in culture solution experiments with higher plants. Am J Bot 25, 322-325

Chen WX, Yan GH, Li JL (1988) Numerical taxonomic study of fast-growing soybean rhizobia and a proposal that Rhizobium fredii be assigned to Sinorhizobium gen nov. Int $J$ Syst Bacteriol 38, 392-397

Cleyet-Marel JC (1987) Dynamique des populations de Rhizobium et de Bradyrhizobium dans le sol et la rhizosphère. Thèse de doctorat d'État ès Sciences. Université Claude-Bernard, Lyon I, $240 \mathrm{p}$

Duong TP, Diep CN (1986) An inexpensive cultural system using ash for cultivation of soybean (G/ycine max L Merrill) on acid clay soils. Plant Soil 96, 225-237

Duong TP, Diep CN, Khiem NT, Hiep NH, Toi NV, Lich NV, Nhan LTK (1984a) Rhizobium inoculant for soybean (Glycine max L Merill) in Mekong delta. II. Response of soybean to Rhizobium inoculation. Plant Soil 79, 235-240

Duong TP, Diep CN, Khiem NT, Hiep NH, Toi NV, Lich NV, Nhan LTK (1984b) Rhizobium inoculant for soybean (Glycine max L Merrill) in Mekong delta. II. Response of soybean to chemical nitrogen fertilizer and Rhizobium inoculation. Plant Soil 79, 241-247

Evans LS, Lewin KF, Vella FA (1980) Effect of nutrient medium $\mathrm{pH}$ on symbiotic nitrogen fixation by Rhizobium leguminosarum and Pisum sativum. Plant Soil 56, 71-80

Keyser HH, Bohlool BB, Hu TS, Weber DF (1982) Fast-growing rhizobia isolated from root nodules of soybean. Science 215, 1631-1632 
Latham M, Kilian J, Pieri C (1984) Fertilité des sols tropicaux. Cah ORSTOM, Sér Pédol 21, 33-41

Lie TA (1969) The effect of low pH on different phases of nodule formation in pea plants. Plant Soil 31, 391-406

Mengel DB, Kamprath EJ (1978) Effect of soil pH and liming on growth and nodulation of soybeans in histosols. Agron J 70, 959-963

Munns DN, Hohneberg JS, Righetti TL, Lauter DJ (1981) Soil acidity tolerance of symbiotic and nitrogen-fertilized soybeans. Agron J 73, 407-410

Robertson JG, Taylor MP (1973) Acid and alkaline invertases in roots and nodules of Lupinus angustifolius infected with Rhizobium lupini. Planta 112, 1-6

Sanchez PA, Villachica JH, Bandy DE (1983) Soil fertility dynamics after clearing a tropical rain forest in Peru. Soil Sci Soc Am J 47, 1171-1178
Scholla MH, Elkan GH (1984) Rhizobium fredii sp nov, a fast-growing species that effectively nodulates soybeans. Int J Syst Bacterio/ 34, 484-486

Schwartz D (1963) Méthodes statistiques à l'usage des médecins et des biologistes. Flammarion Médecine Sciences, Paris, $318 \mathrm{p}$

Thornton FC, Davey CB (1983) Response of the clover-Rhizobium symbiosis to soil acidity and Rhizobium strain. Agron J 75, 557-560

Umbreit WW, Burris RH, Staufer JF (1964) Manometric techniques. Burgess Publ Co, Minneapolis, 4th edn

Young CC, Chang JY, Chao CC (1988) Physiological and symbiotic characteristics of Rhizobium trifolii isolated from subtropical-tropical soils. Biol Fertil Soils 5, 350-354 